\title{
Asparagine-473 Residue Is Important to the Efficient Function of Human Dihydrolipoamide Dehydrogenase
}

\author{
Hakjung Kim* \\ Department of Chemistry, College of Natural Science, Daegu University, Kyoungsan 712-714, Korea
}

Received 27 October 2004, Accepted 15 December 2004

\begin{abstract}
Dihydrolipoamide dehydrogenase (E3) catalyzes the reoxidation of dihydrolipoyl moiety of the acyltransferase components of three $\alpha$-keto acid dehydrogenase complexes and of the hydrogen-carrier protein of the glycine cleavage system. His-457 of Pseudomonas putida E3 is suggested to interact with the hydroxyl group of Tyr-18 of the other subunit and with Glu-446, a component in the last helical structure. To examine the importance of the suggested interactions in human E3 function, the corresponding residue of human E3, Asn-473, was substituted to Leu using site-directed mutagenesis. The E3 mutant was expressed in Escherichia coli and highly purified using an affinity column. Its E3 activity was decreased about 37 -fold, indicating that Asn473 residue was important to the efficient catalytic function of human E3. Its slightly altered spectroscopic properties implied that small conformational changes could occur in the $\mathrm{E3}$ mutant.
\end{abstract}

Keywords: Dihydrolipoamide dehydrogenase, $\alpha$-keto acid dehydrogenase complex, Pyridine nucleotide-disulfide oxidoreductase family, Site-directed mutagenesis, Structural homology

\section{Introduction}

Dihydrolipoamide dehydrogenase (E3) (dihydrolipoamide:NAD ${ }^{+}$ oxidoreductase; EC 1.8.1.4) is a homodimeric enzyme containing one FAD as a prosthetic group at each subunit. Each subunit of human E3 consists of 474 amino acids with a molecular mass of 50,216 daltons calculated from the primary amino acid sequence (Pons et al., 1988). It is present as a common component in three $\alpha$-keto acid dehydrogenase complexes (pyruvate, $\alpha$-ketoglutarate and branched-chain $\alpha$ -

*To whom correspondence should be addressed.

Tel: 82-53-850-6447; Fax: 82-53-850-6449

E-mail: hjkim@daegu.ac.kr keto acid dehydrogenase complexes) (Reed, 1974) and the glycine cleavage system (Walker and Oliver, 1986). It catalyzes the reoxidation of the dihydrolipoyl prosthetic group attached to the lysyl residue(s) of the acyltransferase components of the three $\alpha$-keto acid dehydrogenase complexes and to the hydrogen-carrier protein of the glycine cleavage system.

E3 along with glutathione reductase (GR), thioredoxin reductase, mercuric reductase and trypanothione reductase belong to the pyridine nucleotide-disulfide oxidoreductase family (William, 1976). All of them have homodimeric structures containing an active disulfide center and a FAD in their each subunit. Through the FAD and active disulfide center, they catalyze electron transfers between pyridine nucleotides $\left(\mathrm{NAD}^{+}\right.$or NADPH) and their specific substrates.

The structure of the human E3 active site has been proposed (Jentoft et al., 1992) on the basis of the threedimensional structures of human GR (Thieme et al., 1981) and of Azotobacter vinelandii E3 (Schierbeek et al., 1989). The cDNA sequence for human E3 has been cloned (Pons et al., 1988) and expressed in E. coli (Kim et al., 1991). The recombinant human E3 has possessed similar properties to those of purified mammalian E3s. The putative essential amino acid residues of human E3 have been modified using site-directed mutagenesis and the mutants have been characterized (Kim and Patel, 1992; Leu et al., 1995; Kim, 2002).

The crystal structure of Pseudomonas putida E3 Val shows that the last five amino acid residues form an arm which is not observed in human GR (Mattevi et al., 1992). The removal of the corresponding last five residues in human E3 resulted in a very low E3 activity and instability. This indicated that the last five amino acid residues were essential to the catalytic function and stable structure of human E3 (Kim, 1999b).

His-457, a component in the last helical structure, of Pseudomonas putida E3 is suggested to interact with the hydroxyl group of Tyr-18 of the other subunit and with Glu446 (Mattevi et al., 1992). The corresponding residue, Asn473, of human E3 was substituted to Leu. The E3 mutant 
showed about 37-fold decreased E3 activity and slightly altered spectroscopic properties.

\section{Materials and Methods}

Materials. The electrophoresis reagents, imidazole, iminodiacetic acid sepharose 6B, lipoamide and $\mathrm{NAD}^{+}$were from Sigma Chemical Co. (St. Louis, USA). Dihydrolipoamide was synthesized by reduction of lipoamide using sodium borohydride. Isopropyl- $\beta$ D-thiogalactopyranoside (IPTG) was from POSCOCHEM R\&D Center (Pohang, Korea). E. coli XL1-Blue containing a human E3 expression vector pPROEX-1:E3 was a generous gift from Dr. Mulchand S. Patel of State University of New York at Buffalo. Vent polymerase and T4 DNA ligase were from New England Biolab (Beverly, USA). Primers and dNTP were from Bioneer (Cheongwon, Korea).

Site-directed mutagenesis and construction of the human E3 mutant expression vector pPROEX-1:E3 (N-473->L) Polymerase chain reaction (PCR) was performed with $5^{\prime}$ primer (5'-TCCCAAC GACCGAAAACCTG-3') and 3' mutagenic primer (5'-GCATGC CTCGAGGGA_ACCTCAAAAGAGGATTGAT-3': mismatched bases are underlined and $\mathrm{XhoI}$ sequence is highlighted with bold letters). The reaction was carried out with Vent polymerase in a programmable PCR machine using the human E3 expression vector pPROEX-1:E3 as a template (Khumthong et al., 2002; Kim et al., 2002; Park and Cho, 2002). After denaturation of the template DNA at $95^{\circ} \mathrm{C}$ for $2 \mathrm{~min}, 33$ rounds of temperature cycling were performed at $95^{\circ} \mathrm{C}$ for $30 \mathrm{~s}, 43^{\circ} \mathrm{C}$ for $1 \mathrm{~min}, 72^{\circ} \mathrm{C}$ for $90 \mathrm{~s}$ and a final 7-min incubation at $72^{\circ} \mathrm{C}$ was followed. The PCR generated a 1478-bp DNA fragment containing human E3 sequence of which a codon (AAC) for Asn-473 was substituted to a codon (CTC) for Leu. The fragment was digested with $E c o R I$ and $X h o I$ to generate a 519-bp EcoRI/XhoI fragment. The EcoRI/XhoI fragment was ligated with pPROEX-1:E3 of which the corresponding normal EcoRI/XhoI fragment had been removed by EcoRI and XhoI digestions. The ligation resulted in the construction of the human E3 mutant expression vector pPROEX-1:E3(N-473->L). The mutation was confirmed by DNA sequencing.

Expression and purification of the human E3 mutant One ml of an overnight culture of $E$. coli XL1-Blue containing the mutant expression vector was used to inoculate $200 \mathrm{ml}$ of LB medium containing ampicilin $(100 \mu \mathrm{g} / \mathrm{ml})$. Cells were grown at $37^{\circ} \mathrm{C}$ to an absorbance of 0.7 at $595 \mathrm{~nm}$ and IPTG was added to a final concentration of $1 \mathrm{mM}$ (Kim, 1999a). The growing temperature was shifted to $30^{\circ} \mathrm{C}$ and cells were allowed to grow overnight. The overnight culture was harvested by centrifugation at $4,000 \times g$ for 5 min. Cell pellets were washed with $50 \mathrm{mM}$ potassium phosphate buffer (pH 8.0) containing $100 \mathrm{mM} \mathrm{NaCl}$ (Binding buffer) and then recollected by centrifugation at $4,000 \times g$ for $5 \mathrm{~min}$. They were resuspended in $10 \mathrm{ml}$ of Binding buffer. They were lysed by a sonication treatment and centrifuged at $10,000 \times g$ for $20 \mathrm{~min}$. The supernatant was loaded on a nickel iminodiacetic acid sepharose $6 \mathrm{~B}$ column. The column was washed with 10 column volumes of Binding buffer and then with the same volume of Binding buffer

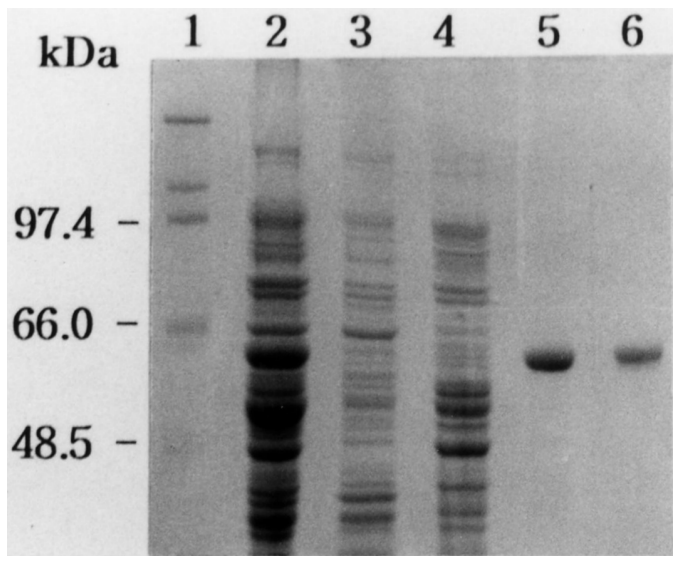

Fig. 1. SDS-polyacrylamide gel for the purification of the human E3 mutant. Lane 1, molecular weight marker (from bottom to top, bovine erythrocytes carbonic anhydrase, porcine heart fumarase, bovine serum albumin, rabbit muscle phosphorylase $b$; lane 2, supernatant; lane 3, flow-through; lane 4, Binding buffer containing $150 \mathrm{mM}$ imidazole; lane 5, Binding buffer containing $500 \mathrm{mM}$ imidazole; lane 6 , previously purified normal recombinant human E3 as a control.

containing $150 \mathrm{mM}$ imidazole. The E3 mutant was eluted with Binding buffer containing $500 \mathrm{mM}$ imidazole. The purification steps were analyzed by the SDS-polyacrylamide gel electrophoresis as shown in Fig. 1.

E3 assay and spectroscopic study E3 activity was assayed at $37^{\circ} \mathrm{C}$ in $50 \mathrm{mM}$ potassium phosphate buffer $(\mathrm{pH} 8.0$ ) containing 1.5 mM EDTA. The activity was recorded spectrophotometrically by observing the reduction of $\mathrm{NAD}^{+}$at $340 \mathrm{~nm}$ with a Shimazu UV $160 \mathrm{~A}$ recording spectrophotometer. One unit of activity is defined as $1 \mathrm{~mol}$ of $\mathrm{NAD}^{+}$reduced per min/mg of protein at $37^{\circ} \mathrm{C}$.

The UV-visible absorption spectrum was recorded using the Shimazu UV 160A recording spectrophotometer from $200 \mathrm{~nm}$ to $650 \mathrm{~nm}$. The fluorescence spectra were recorded using a Fluoromax spectrofluorometer (Industries Inc., Edison, NJ, USA). E3 was excited at $296 \mathrm{~nm}$ and the emission was recorded from $302 \mathrm{~nm}$ to $580 \mathrm{~nm}$. The data were transferred to an ASCII file and the spectra were drawn using the MicroCal Origin program (Photon Technology International, South Brunswick, NJ, USA).

\section{Results and Discussion}

The carboxy-terminus regions of E3s from several sources and human GR are shown in Table 1. The crystal structure of $P$. putida E3 Val shows that the last five amino acids form an arm (Mattevi et al., 1992). GR, having a good structural homology to E3s, lacks the corresponding carboxy-terminus region. The removal of the corresponding last five amino acids in human E3 led to a very low E3 activity $(0.17 \%)$ and altered spectroscopic properties (Kim, 1999b). These results indicated that the last five amino acids were critical to the catalytic function and stable structure of human E3. 
Table 1. Comparison of amino acid sequences at carboxyterminus region of $\mathrm{E} 3 \mathrm{~s}$ from several sources and human glutathione reductase (GR). The Asn-473 residue in human E3 and the corresponding residues in other E3s are highlighted by italic bold letters

\begin{tabular}{cll}
\hline Protein & \multicolumn{1}{c}{ Source } & \multicolumn{1}{c}{ Amino acid sequence } \\
\hline E3 & Pseudomonas putida & 446-EAALRALGHALHI-458 \\
E3 & Yeast & 466-ANMAAYD-KAIHC-478 \\
E3 & Pig & 462-ANLAASFGKAINF-474 \\
E3 & Human & 462-ANLAASFGKSINF-474 \\
GR & Human & 477-LR-478 \\
\hline
\end{tabular}

His-457 of $P$. putida E3 Val, a component of the last five amino acids, has been suggested to be important in the structure and function of the enzyme by forming a weak hydrogen bonding with the hydroxyl group of Tyr-18 of the other subunit (Mattevi et al., 1992). It is also suggested to interact with Glu-446, a component in the last helical structure. To examine the importance of the suggested interactions in human E3 function, the corresponding residue in human E3, Asn-473, was substituted to Leu having a similar occupying volume. The substitution was expected to destroy the ability of Asn-473 forming the presumed hydrogen bonding interactions.

Site-directed mutagenesis and construction of the mutant expression vector were performed as described in Materials and Methods. The PCR generated a $1478 \mathrm{bp}$ E3 DNA fragment in which codon (AAC) for Asn-473 was substituted to a codon (CTC) for Leu. The fragment was digested with EcoRI and XhoI and the resulting $519 \mathrm{bp} \mathrm{EcoRI/XhoI}$ fragment with the mutations was isolated by agarose gel electrophoresis. The human E3 expression vector pPROEX1:E3 was digested with EcoRI and XhoI to remove the corresponding normal EcoRI/XhoI fragment. The resulting human E3 expression vector lacking the normal EcoRI/XhoI fragment was isolated by agarose gel electrophoresis. The isolated vector was ligated with the previously isolated EcoRI/ XhoI fragment, resulting in the construction of the mutant expression vector pPROEX-1:E3(N-473->L). This process also removed the pre-existing BamHI site. The removal of the BamHI site was used for the screening of the mutant expression vector. The mutations were also confirmed by DNA sequencing.

The expression and purification of the mutant were performed as described in Materials and Methods. After equilibration with Binding buffer, the supernatant solution was applied to the column. E. coli proteins were washed out with Binding buffer and then with Binding buffer containing 150 $\mathrm{mM}$ imidazole. Since E3 contains FAD as a prosthetic group, it has a bright yellow color. The bright yellow color became clearer as E. coli proteins were washed out. This color was a good indicator for the migration of the mutant through the column. The mutant was eluted with Binding buffer containing $500 \mathrm{mM}$ imidazole. Purification was followed by

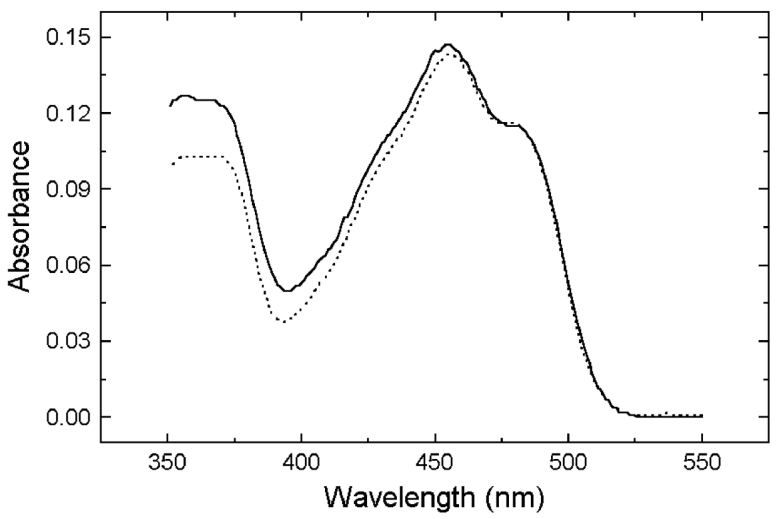

Fig. 2. UV-visible spectra of the Leu- 473 mutant $(13.0 \mu \mathrm{M}$, solid line) and normal $(12.5 \mu \mathrm{M}$, dot line) recombinant human E3s. The spectra were recorded using Shimazu UV 160A spectrophotometer and the data from $350 \mathrm{~nm}$ to $550 \mathrm{~nm}$ were transferred to an ASCII file and the spectra were drawn using the MicroCal Origin program.

SDS-polyacrylamide gel electrophoresis as shown in Fig. 1. The gel showed that the E3 mutant was highly purified.

The E3 activity of the mutant was determined as 16.6 unit/ $\mathrm{mg}$ at substrate concentrations of $2 \mathrm{mM}$ dihydrolipoamide and $3 \mathrm{mM} \mathrm{NAD}^{+}$at $37^{\circ} \mathrm{C}$. This value was about 37 -fold lower than that of normal human E3 activity determined at the same condition. Even though it was difficult to obtain the detailed kinetic parameters due to the low activity and instability of the mutant, this 37-fold decreased E3 activity of the Leu-473 mutant clearly indicated that the Asn-473 residue of human E3 was important to the efficient catalytic function of the enzyme.

To examine any conformational changes in the mutant due to the substitution, UV-visible absorption and fluorescence spectra were observed as described in Materials and Methods. Human E3 contains one FAD as a prosthetic group in each subunit. It, therefore, shows a characteristic UV-visible absorption spectrum of flavoproteins as shown in Fig. 2 (dot line). It has two peaks, one broad peak from $358 \mathrm{~nm}$ to 370 $\mathrm{nm}$ and the other at $455 \mathrm{~nm}$. There is a unique shoulder between $465 \mathrm{~nm}$ and $485 \mathrm{~nm}$ which has been observed in many flavoproteins. The strong association of FAD with the flavoproteins results in these characteristic features in the spectrum. The overall shape of the mutant spectrum (solid line) was similar to that of the normal recombinant human E3 spectrum (dot line). However, an alteration was found in the region of the peak $(358 \mathrm{~nm}$ to $370 \mathrm{~nm})$. The absorption intensity of the E3 mutant in this region was slightly higher than that of the normal enzyme. This difference in the absorption spectra implied that small conformational changes could occur in the mutant. The changes could affect the association of FAD with the enzyme so that the alteration at the peak ( $358 \mathrm{~nm}$ to $370 \mathrm{~nm}$ ) in the UV-visible spectrum of the mutant could occur.

Another evidence for the small conformational changes in 


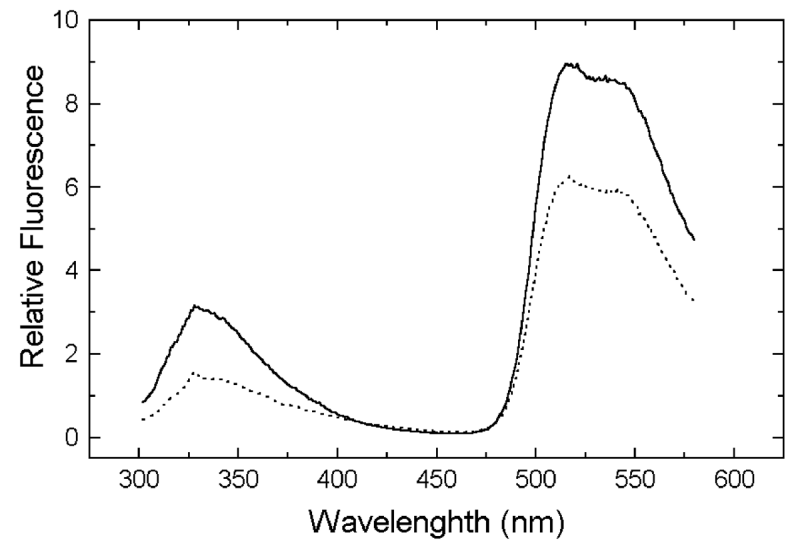

Fig. 3. Fluorescence spectra of the Leu- 473 mutant $(13.0 \mu \mathrm{M}$, solid line) and normal $(12.5 \mu \mathrm{M}$, dot line) recombinant human E3s. Enzymes were excited at $296 \mathrm{~nm}$ and the emissions were observed from $302 \mathrm{~nm}$ to $580 \mathrm{~nm}$. The data were transferred to an ASCII file and the spectra were drawn using the MicroCal Origin program.

the mutant came from the fluorescence study, which was very sensitive to any structural changes in proteins. E3s were excited at $296 \mathrm{~nm}$ and the fluorescence emissions were observed from $302 \mathrm{~nm}$ to $580 \mathrm{~nm}$. As shown in Fig. 3, two fluorescence emissions were observed for both E3s. The first emission from $300 \mathrm{~nm}$ to $400 \mathrm{~nm}$ was due to aromatic amino acids, mainly tryptophans. The second emission from $480 \mathrm{~nm}$ to over $550 \mathrm{~nm}$ was due to FAD. When the fluorescence spectra of E3s were compared, a noticeable difference was found in the ratio between relative intensities of the first and second fluorescence emissions. The ratio (about 3) between relative intensities of the first and second fluorescence emissions of the mutant (solid line) was slightly lower than that (about 4) of the normal enzyme (dot line). This difference in the fluorescence spectra implied again that the small conformational changes could occur in the mutant. The substitution of Asn-473 to Leu could also provide more hydrophobic environments in the active site region of the mutant. This more hydrophobic environment might also partly contribute to the slightly changed fluorescence spectrum pattern of the mutant.

From these results, the following suggestions can be made. First, the Asn-473 residue is important to the efficient function of human E3, by presumably making similar hydrogen bonding interactions with the neighboring amino acids as observed in the three-dimensional structure of $P$. putida E3 Val. Second, the removal of these interactions by the substitution of Asn-473 to Leu could cause the small conformational changes in human E3.

Acknowledgments The author thanks Dr. Mulchand S. Patel (State University of New York, Buffalo) for a generous gift of an E. coli XL1-Blue containing a human E3 expression vector. The author is grateful to Dr. Tai Jong Kang (Daegu
University) for providing a fluorometer. This research was supported in part by the Daegu University Research Grant, 2003.

\section{References}

Jentoft, J. E., Shoham, M., Hurst, D. and Patel, M. S. (1992) A structural model for human dihydrolipoamide dehydrogenase. Proteins 14, 88-101.

Khumthong, R., Angsuthanasombat, C., Panyim, S. and Katzenmeier, G. (2002) In vitro determination of Dengue virus type 2 NS2B-NS3 protease activity with fluorescent peptide substrates. J. Biochem. Mol. Biol. 35, 206-212.

Kim, H (1999a) Protein engineering of an artificial intersubunit disulfide bond linkage in human dihydrolipoamide dehydrogenase. J. Biochem. Mol. Biol. 32, 76-81.

Kim, H (1999b) Deletion of the last five amino acid residues in human dihydrolipoamide dehydrogenase. Bull. Korean Chem. Soc. 20, 1221-1224.

Kim, H (2002) Activity of human dihydrolipoamide dehydrogenase is reduced by mutation at threonine- 44 of FAD-binding region to Valine. J. Biochem. Mol. Biol. 35, 437-441.

Kim, Y-S., Ha, K-S., Kim, Y.-H. and Bae, Y.-S. (2002) The ring$\mathrm{H} 2$ finger motif of CKBBP1/SAG is necessary for interaction with protein kinase CKII and optimal cell proliferation. $J$. Biochem. Mol. Biol. 35, 629-636.

Kim, H., Liu, T.-C. and Patel, M. S. (1991) Expression of cDNA sequences encoding mature and precursor forms of human dihydrolipoamide dehydrogenase in Escherichia coli. J. Biol. Chem. 266, 9367-9373.

Kim, H. and Patel, M. S. (1992) Characterization of two sitespecifically mutated human dihydrolipoamide dehydrogenases (His-452 $\rightarrow$ Gln and Glu-457 $\rightarrow$ Gln). J. Biol. Chem. 267, 5128-5132.

Liu, T., Korotchkina, L. G., Hyatt, S. L., Vettakkorumakankav, N. N. and Patel, M. S. (1995) Spectroscopic studies of the characterization of recombinant human dihydrolipoamide dehydrogenase and its site-directed mutants. J. Biol. Chem. 270, 15545-15550.

Mattevi, A., Obmolova, G., Sokatch, J. R., Betzel, C. and Hol, W. G. J. (1992) The refined crystal structure of Pseudomonas putide lipoamide dehydrogenase complexed with $\mathrm{NAD}^{+}$at 2.45 A resolution. Proteins 13, 336-351

Park, S-J and Cho, Y-D. (2002) Structural roles of cysteine 50 and cysteine 230 residues in Arabidopsis thaliana S-adenosylmethionine decarboxylase. J. Biochem. Mol. Biol. 35, 178-185.

Pons, G., Raefsky-Estrin, C., Catothers, D. J., Pepin, R. A., Javed, A. A., Jesse, B. W., Ganapathi, M. K., Samols, D. and Patel, M. S. (1988) Cloning and cDNA sequence of the dihydrolipoamide dehydrogenase component of human aketoacid dehydrogenase complexes. Proc. Natl. Acad. Sci. USA 85, 1422-1426.

Reed, L. J. (1974) Multienzyme complexes. Acc. Chem. Res. 7, 40-46.

Schierbeek, A. J., Swarte, M. B. A., Dijkstra, B. W., Vriend, G., Reed, R. J., Hol, W. G. J. and Drenth, J. (1989) X-ray structure of lipoamide dehydrogenase from Azotobacter vinelandii determined by a combination of molecular and 
isomorphous replacement techniques. J. Mol. Biol. 206, 365379.

Thieme, R., Pai, E. F., Schirmer, R. H. and Schulz, G. E. (1981) Three-dimensional structure of glutathione reductase at $2 \AA$ resolution. J. Mol. Biol. 152, 763-782.

Walker, J. L. and Oliver, D. J. (1986) Glycine decarboxylase multienzyme complex. Purification and partial characterization from leaf mitochondria. J. Biol. Chem. 261, 2214-2221.

Williams, C. H., Jr. (1976) Flavin containing dehydrogenases; in Enzymes, Boyer, P. (ed.), pp. 89-173, Academic Press, New York, USA. 